\title{
Stability of an Analog Optimization Circuit for Quadratic Programming
}

\author{
Sergey Vichik, Murat Arcak, Francesco Borrelli \\ University of California, Berkeley, 94720, USA, \{sergv,arcak,fborrelli\}@berkeley.edu. \\ Submitted to: Systems $\&$ Control Letters
}

\begin{abstract}
We study the stability of an analog optimization circuit that solves quadratic programming (QP) problems. The circuit dynamics are modeled as a switched affine system. A piece-wise quadratic Lyapunov function and the KYP lemma are used to derive the stability criterion. The stability criterion characterizes the range of critical circuit parameters for which the QP circuit is globally exponentially stable.
\end{abstract}

Keywords: optimization, MPC, quadratic programming, analog computation, KYP, Lyapunov function, hybrid systems

\section{Introduction}

Our renewed interest in analog optimization stems from the need to achieve a low latency solution for Model Predictive Control (MPC) [6]. In MPC at each sampling time, starting at the current state, an open-loop optimal control problem is solved over a finite horizon. The optimal command signal is applied to the process only during the following sampling interval. At the next time step a new optimal control problem based on new measurements of the state is solved over a shifted horizon. The optimal solution relies on a dynamic model of the process, respects input and output constraints, and minimizes a performance index. When the model is linear and the performance index is based on the two-norm, the resulting optimization problem can be cast as a quadratic program (QP), where the state enters the right hand side (rhs) of the constraints.

In [17] we presented the design of an analog QP circuit. We showed that its equilibrium voltages are the QP optimizers. The proposed circuit achieves lower latency and is simpler than the designs in the early analog optimization work in $[4,16,10]$.

In this paper we study the dynamics of the circuit. Circuits that combine linear dynamics and switching elements have been extensively studied in the past $[13,8]$. We describe the circuit as continuous-time piecewise affine system with restricted switching logic and we derive a criterion for the exponential stability using a piecewise quadratic Lyapunov function. Stability of a piecewise affine system can be shown by numerically solving an appropriate $\operatorname{LMI}[9,7,5,13]$. In this paper we exploit the structure of the circuit to show that the Lyapunov function exists for a range of critical circuit parameters. We make use of an eigenvalue decomposition and KYP lemma to derive the circuit parameter bound. Our results allow to quantify the maximum circuit speed as a function of the circuit parasitic effects.

Although we address a particular system in this paper, the methodology is of broader interest. This is because the resistor network in our system exhibits the general structure of diffusive coupling - an area of extensive research activity in dynamical systems. In addition, our system has particular features, such as state dependent switching of the coupling and nonuniform bias terms affecting the subsystems. Our methodology accounts for these features and may prompt further research for broader diffusively coupled systems with similar characteristics.

The paper is organized as follows. For the sake of better readability, a description of how to construct an analog circuit from a given QP is presented in Section 2. For more details and experimental results we refer the reader to the description in [17]. Section 3 presents stability analysis of the circuit and the main result of the paper. Concluding remarks are presented in Section 4.

\section{QP Analog Circuit}

Consider the quadratic programming (QP) problem

$$
\begin{array}{r}
\min _{V=\left[V_{1}, \ldots, V_{n}\right]^{T}} V^{T} Q V \\
\text { s.t. } \quad A_{\text {eq }} V=b_{\text {eq }} \\
A_{\text {ineq }} V \leq b_{\text {ineq }}
\end{array}
$$

where $V_{1}, \ldots, V_{n}$ are the optimization variables, $b_{\text {eq }}$ and $b_{\text {ineq }}$ are column vectors, $Q \succ 0$, and $A_{\text {ineq }}$ and $A_{\text {eq }}$ are matrices.

Without loss of generality, we assume that $A_{\text {ineq }}$ and $A_{\text {eq }}$ have non-negative entries. Indeed, a QP (1) with negative entries can be transformed into this form by intro- 


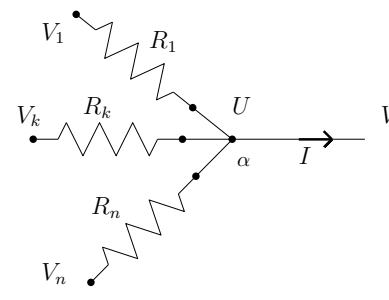

(a)

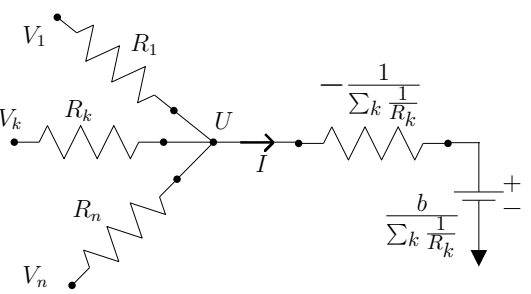

(b)
Figure 1: (a) A node with $k$ connected wires. (b) Equality enforcing circuit consisting of $n$ resistors $R_{k}$, a negative resistance and a reference voltage.

ducing an auxiliary vector $\bar{V}$ as follows:

$$
\begin{array}{ll}
\min _{\bar{V}, V} & V^{T} Q V \\
\text { s.t. } & A_{\text {eq }}^{+} V+A_{\text {eq }}^{-} \bar{V}=b_{\text {eq }}, A_{\text {ineq }}^{+} V+A_{\text {ineq }}^{-} \bar{V} \leq b_{\text {ineq }} \\
& V+\bar{V}=0
\end{array}
$$

where $A_{\text {ineq }}$ and $A_{\text {eq }}$ are split into positive and negative parts $\left(A_{\text {ineq }}=A_{\text {ineq }}^{+}-A_{\text {ineq }}^{-}\right.$and $\left.A_{\text {eq }}=A_{\text {eq }}^{+}-A_{\text {eq }}^{-}\right)$.

In the beginning of this section we present the basic building blocks which will be later used to create a circuit that solves problem (1). The first basic block enforces equality constraints of the form (1b). The second building block enforces inequality constraints of the form (1c). The last basic block implements the cost function.

\subsection{Equality constraint}

Consider the circuit depicted in Fig. 1a. In this circuit $n$ wires are connected to a common node. We call this common node $\alpha$, its potential $U$ and the current that exits this node $I$. Kirchhoff's current law (KCL) implies

$$
\sum_{k=1}^{n} I_{k}=\sum_{k=1}^{n} \frac{V_{k}-U}{R_{k}}=I
$$

where $V_{k}$ is the potential of node $k, R_{k}$ is the resistance between node $k$ and the node $\alpha$. Equation (3) can be written as an equality constraint on potentials $V_{k}$ :

$$
\sum_{k=1}^{n} \frac{V_{k}}{R_{k}}=I+U \sum_{k=1}^{n} \frac{1}{R_{k}}
$$

If we can set the right hand side (rhs) of (4) to any desired value $b$, then (4) enforces an equality constraint on a linear combination of $V_{k}$ variables. Therefore, every equality constraint $(1 \mathrm{~b})$ can be implemented by assigning

$$
U=\frac{b-I}{\sum_{k=1}^{n} \frac{1}{R_{k}}} .
$$

Equation (5) together with (4) yields

$$
\left[\begin{array}{ccc}
\frac{1}{R_{1}} & \ldots & \frac{1}{R_{n}}
\end{array}\right]\left[\begin{array}{c}
V_{1} \\
\vdots \\
V_{n}
\end{array}\right]=b
$$

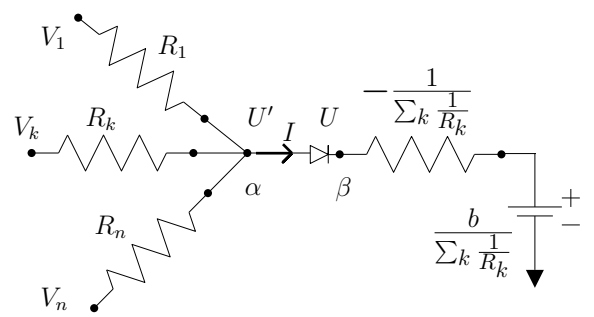

(a)

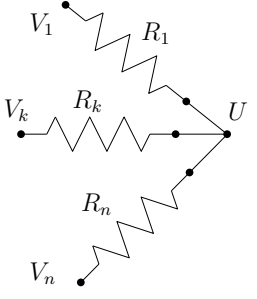

(b)
Figure 2: (a) Inequality enforcing circuit. (b) Quadratic cost circuit.

and the circuit implementing (6) is shown in Fig. $1 \mathrm{~b}$.

\subsection{Inequality constraint}

Consider the circuit shown in Fig. 2a. Similarly to the equality constraint circuit, $n$ wires are connected to a common node $\alpha$. Its potential is $U^{\prime}$ and the current exiting this node is $I$. Kirchhoff's current law (KCL) implies (3).

An ideal diode connects node $\alpha$ to node $\beta$. The potential of node $\beta$ is $U$. The diode enforces $U^{\prime} \leq U$. In Fig. 2a, the voltage $U$ can be computed as follows

$$
U=\frac{b-I}{\sum_{k=1}^{n} \frac{1}{R_{k}}} \geq U^{\prime}
$$

Equation (3) and $U^{\prime} \leq U$ yield

$$
\sum_{k=1}^{n} \frac{V_{k}}{R_{k}}=I+U^{\prime} \sum_{k=1}^{n} \frac{1}{R_{k}} \leq I+U \sum_{k=1}^{n} \frac{1}{R_{k}}=b
$$

which can be compactly rewritten as

$$
\left[\begin{array}{lll}
\frac{1}{R_{1}} & \cdots & \frac{1}{R_{n}}
\end{array}\right]\left[\begin{array}{c}
V_{1} \\
\vdots \\
V_{n}
\end{array}\right] \leq b .
$$

\subsection{Quadratic cost function}

Let $A=\left[\begin{array}{c}A_{\text {eq }} \\ A_{\text {ineq }}\end{array}\right]$ be the matrix of constraint coefficients. By composing the elementary circuits of the previous section we can design an analog circuit which implements the constraints $A_{\text {eq }} V=b_{\text {eq }}$ and $A_{\text {ineq }} V \leq b_{\text {ineq }}$. By using simple energy arguments, in [17] it was shown that such circuit would minimize a cost function $V^{T} Q_{A} V$ where

$$
Q_{A}=\operatorname{diag}\left(\mathbf{1}^{T} A\right)-A^{T} \operatorname{diag}\left(\mathbf{1}^{T} A^{T}\right)^{-1} A .
$$

In general, this cost function is different from the desired cost $Q$. However, it is possible to add redundant constraints of the form $A_{\text {augm }} V<\infty$, which are always inactive and have no effect on a feasible set of the problem (1). By doing so the cost matrix can be shaped in a way that $Q_{A^{\prime}}=k Q$, where $A^{\prime}=\left[\begin{array}{c}A \\ A_{\text {augm }}\end{array}\right], k>0$ is a scalar, and 


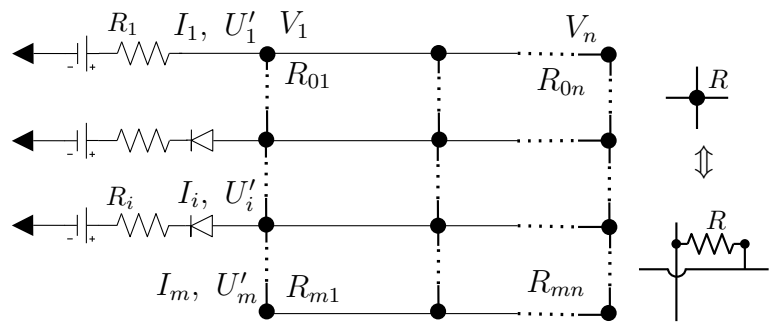

Figure 3: Analog circuit solving a QP. Vertical wires are variable nodes with potentials $V_{1} \ldots V_{n}$. Black dots represent resistances that connects vertical and horizontal wires. Horizontal wires are cost or constraint nodes. Some of the horizontal wires are connected to a ground via a negative resistance $R_{i}=-\left(\sum_{j} 1 / R_{i j}\right)^{-1}$, a constant voltage source and a diode for inequality nodes.

$Q_{A^{\prime}}=\operatorname{diag}\left(\mathbf{1}^{T} A^{\prime}\right)-A^{\prime T} \operatorname{diag}\left(\mathbf{1}^{T} A^{\prime T}\right)^{-1} A^{\prime}$ (see [17] for additional details). The redundant constraints are implemented using a simple circuit depicted in Fig. 2b, i.e, a special case of the inequality circuit, without the diode and the negative resistor.

\subsection{Connecting the basic circuits}

This section presents how to construct the circuit that solves a general QP. We construct the conductance matrix $A \in \mathbb{R}^{m \times n}$ as

$$
A \triangleq\left[\begin{array}{c}
A_{\text {eq }} \\
A_{\text {ineq }}
\end{array}\right]
$$

and denote $A_{i j}$ the $i, j$ element of $A$. For a given QP (1) the $R_{i j}$ resistor is defined as

$$
R_{i j}=\frac{1}{A_{i j}}, i=1, \ldots m, j=1, \ldots, n .
$$

Consider the circuit shown in Fig. 3. The circuit is shown using a compact notation where each resistor $R_{i j}$ is represented by a dot, vertical wires represent variable nodes with potentials $V_{1} \ldots V_{n}$ and horizontal wires represent constraint nodes. The compact representation of a resistor through the dot symbol is clarified in Fig. 3. If $A_{i j}=0$ then no resistor is present in the corresponding dot.

The QP circuit is constructed by connecting the nodes associated with the variables $V_{1} \ldots V_{n}$ to all three types of the basic circuits: equality, inequality and cost. We will refer to such nodes as variable nodes. Each row of the circuit in Fig. 3 is one of the basic circuits presented in Sections 2.1, 2.2 and 2.3.

\subsection{Steady state solution}

Consider the circuit in Fig. 3. Let $U^{\prime}=\left[U_{1}^{\prime}, \ldots, U_{m}^{\prime}\right]^{T}$ be the voltages of the constraint nodes as shown in Fig. 3.
By applying the Kirchhoff's current law we can show [17] that the circuit is characterized by

$$
\begin{aligned}
& A V=\operatorname{diag}\left(\mathbf{1}^{T} A^{T}\right) U^{\prime}+I \\
& A^{T} U^{\prime}=\operatorname{diag}\left(\mathbf{1}^{T} A\right) V \\
& A_{\text {eq }} V=b_{\text {eq }}, A_{\text {ineq }} V \leq b_{\text {ineq }}, \quad I_{\text {ineq }} \geq 0 \\
& {\left[A_{\text {ineq }} V-b_{\text {ineq }}\right]_{i}\left[I_{\text {ineq }}\right]_{i}=0, \forall i \in \mathcal{I},}
\end{aligned}
$$

where $\mathcal{I}$ is the index set of all inequality constraints. In the previous work [17] we proved that the circuit steady state potentials $V_{1} \ldots V_{n}$ (i.e., a solution to equations (13)) are a solution of the QP problem (1).

\section{Stability}

The ideal model of the circuit, as presented in Section 2, does not contain dynamical elements. In practice there will be parasitic effects and time constants associated to the implementation of a negative resistance. Moreover, the combination of a negative resistance and a capacitance is potentially unstable. Therefore, stability of the circuit must be addressed. This section presents the stability analysis of the QP circuit assuming the presence of a parasitic capacitances and the realization of a negative resistance by using an operational amplifier (opamp).

\subsection{Dynamic Model}

The negative resistor element that is required for equality and inequality constraints can be realized using an operational amplifier with resistor feedback, as shown in Fig. 4a. In this circuit a voltage $U$ is applied on an input terminal and the circuit yields a current $I$ (or vice-versa). The two input voltages of an ideal opamp are equal in steady state $\left(U=V_{f}\right)$, and it is immediate to show that $I=-\frac{U}{R}+b$. Thus, the circuit functions as a negative resistance of $-R$.

In summary, we use the representation in Fig. $4 \mathrm{~b}$ of the analog QP circuit and study its stability. The circuit in Fig. $4 \mathrm{~b}$ is obtained from the circuit in Fig. 3 by replacing diodes with switches and using the model of negative resistance as in Fig. 4a. In addition, the capacitances in Fig. $4 \mathrm{~b}$ capture the dominant parasitic effect. We remind the reader that the circuit consists of $m$ negative resistors connected through $m$ switches to a passive resistor network. It models the optimization circuit, where switches can be either always on (equality constraint), or switching on and off (representing a diode for an inequality constraint), or always off (quadratic cost).

We assume that the circuit is scaled in such a way that the sum of each row of the matrix $A$ is the same and equals $R^{-1}$, thus

$$
\operatorname{diag}\left(\mathbf{1}^{T} A^{T}\right)=R^{-1} \mathbb{I}^{m}
$$

where $\mathbb{I}^{m}$ is the identity matrix of size $m$. Any problem can be scaled in this way by multiplying every constraint 


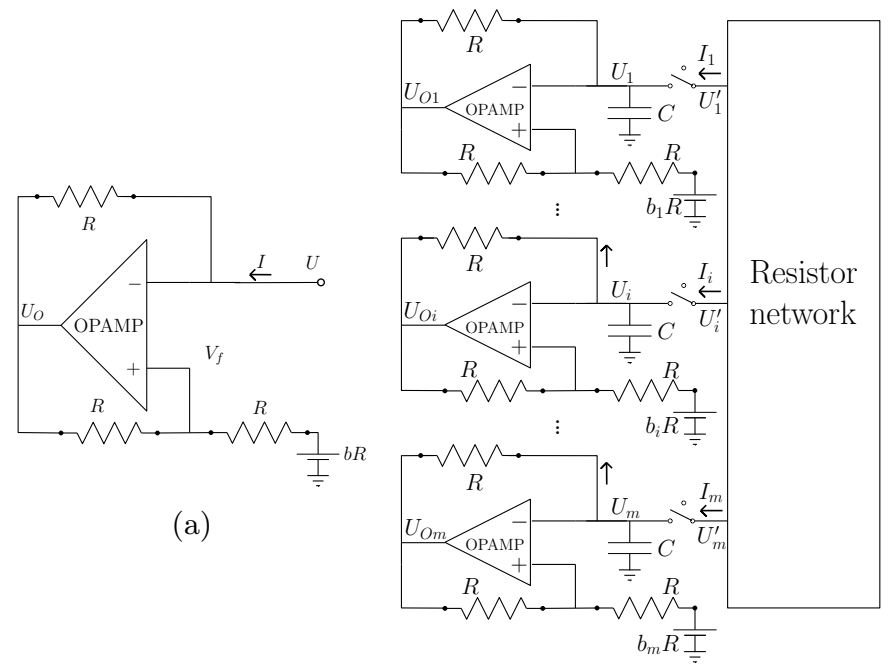

(b)

Figure 4: (a) Realization of negative resistance with an operational amplifier. (b) Circuit model with opamps and parasitic capacitances.

row in (1) by a positive constant. Furthermore, this scaling yields practical benefits by unifying the design of the negative resistors.

We describe the dynamics of an operational amplifier with the first order differential equation

$$
\frac{d U_{O}}{d t}=-\frac{U_{O}}{\tau}+\left(V_{f}-U\right) \frac{K}{\tau},
$$

where $K$ is the gain of an opamp and $\tau$ is the time constant of an opamp. Then, the dynamics of each negative resistance circuit and each parasitic capacitor connected to $U_{i}$ node (as in Fig. $4 \mathrm{~b}$ ) is given by

$$
\begin{array}{ll}
\frac{d U_{i}}{d t}=-\frac{U_{i}-U_{O i}}{C R}+\frac{I_{i}}{C} & i=1, \ldots, m \\
\frac{d U_{O i}}{d t}=U_{O i} \frac{K-2}{2 \tau}-U_{i} \frac{K}{\tau}+\frac{b_{i} R K}{2 \tau} & i=1, \ldots, m,
\end{array}
$$

where $U_{i}$ is the voltage of $i$-th negative resistance, $U_{O i}$ is the output voltage of $i$-th opamp, and $b_{i}$ is the rhs constant from (1). Denoting

$$
\gamma \triangleq \frac{R C K}{\tau}
$$

we rewrite (16) as

$$
\begin{array}{ll}
\frac{d U_{i}}{d t^{\prime}}=-\left(U_{i}-U_{O i}\right)+R I_{i} & i=1, \ldots, m \\
\frac{d U_{O i}}{d t^{\prime}}=\left(\frac{\gamma}{2}-\frac{\gamma}{K}\right) U_{O i}-\gamma U_{i}+\frac{\gamma b_{i} R}{2} & i=1, \ldots, m,
\end{array}
$$

where $t^{\prime} \triangleq t / R C$ is the normalized time. We notice that the steady state solution for $K \rightarrow \infty$ of these equations recovers the ideal negative resistor characteristics $U_{i}-R b_{i}=$ $-R I_{i}$.
The non-dimensional parameter $\gamma$ is the ratio of the circuit $R C$ time constant to an approximated closed loop time constant of the opamp $\tau / K$. We will show that the value of this parameter is critical for the circuit stability and we will show that there exist a range of values that make the circuit stable.

\subsection{Compact Form - Hybrid Model}

Next we rewrite the circuit dynamics in a compact form as a switched affine system. The negative resistor circuits are connected via the resistor network that can be modeled as a coupling matrix. When potentials $U_{1}, \ldots, U_{m}$ are applied to the resistor network as in Fig. 4b the current of every port can be obtained by solving (13b) and (13a). From (13b) we know that $V=\operatorname{diag}\left(\mathbf{1}^{T} A\right)^{-1} A^{T} U^{\prime}$ and substitution into (13a) yields

$$
I=\left(A \operatorname{diag}\left(\mathbf{1}^{T} A\right)^{-1} A^{T}-\operatorname{diag}\left(\mathbf{1}^{T} A^{T}\right)\right) U^{\prime} .
$$

Let $\bar{A} \triangleq \operatorname{diag}\left(\mathbf{1}^{T} A^{T}\right)^{-1} A$ be a normalized conductance matrix, such that sum of each row equals to 1 . Denote normalized current as $\bar{I}=R I$ and after substitution of $\bar{I}$ and $\bar{A}$ in (19) the current voltage relation is written as

$$
\begin{aligned}
& \bar{I}=\overline{\mathcal{A}} U^{\prime} \\
& \overline{\mathcal{A}}=\bar{A} \operatorname{diag}\left(\mathbf{1}^{T} \bar{A}\right)^{-1} \bar{A}^{T}-\mathbb{I}^{m},
\end{aligned}
$$

where $U^{\prime}$ is the vector of voltages $U_{i}^{\prime}$ f applied to the resistor network as in Fig. 4b. If an associated switch $i$ is closed, then $U_{i}=U_{i}^{\prime}$. We want to use only the voltages $U$ in the model and, therefore, need to solve for and substitute voltages $U^{\prime}$ that correspond to open switches.

Define the circuit state vector as

$$
X \triangleq\left[U_{1}, U_{O 1}, \ldots, U_{m}, U_{O m}\right]^{T} .
$$

We divide the state space $\mathbb{R}^{2 m}$ into regions $\mathcal{R}^{v}$ that correspond to a given switch combination. Let $v$ be the integer representation of the binary vector modeling the status of the switches in Fig. $4 \mathrm{~b}$, where $v \in\left\{0, \ldots, 2^{|\mathcal{I}|}-1\right\}$ and $|\mathcal{I}|$ is the number of inequalities in the circuit. Let the matrix $S^{v} \in \mathbb{R}^{m \times m}$ be a permutation matrix that sorts $U$ in a way that the first variables are associated with the closed switches of a given switch combination $v$. Let $m_{c, v}$ be a number of closed and $m_{o, v}$ be a number of open switches. $X_{c}^{v}$ denotes all elements of the vector $X$ corresponding to the closed switches of the switch index $v$. Similarly $X_{o}^{v}$ refers to open switches. With this notation we have

$$
S^{v} U=\left[\begin{array}{c}
U_{c}^{v} \\
U_{o}^{v}
\end{array}\right] \text {. }
$$

The matrix $\overline{\mathcal{A}}$ in (20b) can be partitioned to closed and open switch blocks

$$
\overline{\mathcal{A}}=S^{v,-1}\left[\begin{array}{cc}
\overline{\mathcal{A}}_{c c}^{v} & \overline{\mathcal{A}}_{c o}^{v} \\
\overline{\mathcal{A}}_{o c}^{v} & \overline{\mathcal{A}}_{o o}^{v}
\end{array}\right] S^{v} .
$$




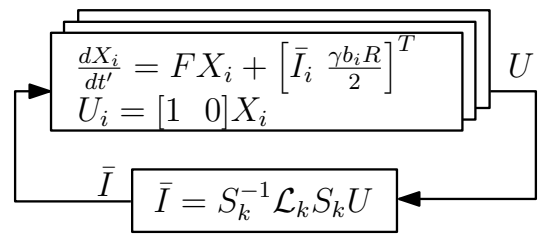

Figure 5: Feedback system interconnection. The coupling matrix represents resistor network that is driven by potentials $U$ applied to its connected ports and its output is the current $\bar{I}$.

For closed switches $U_{c}^{\prime v}=U_{c}^{v}$ and for open switches the current $\bar{I}_{o}^{v}$ must be zero. We use those constraints and substitute (23) into (20)

$$
\left[\begin{array}{c}
\bar{I}_{c}^{v} \\
0
\end{array}\right]=\left[\begin{array}{ll}
\overline{\mathcal{A}}_{c c}^{v} & \overline{\mathcal{A}}_{c o}^{v} \\
\overline{\mathcal{A}}_{o c}^{v} & \overline{\mathcal{A}}_{o o}^{v}
\end{array}\right]\left[\begin{array}{c}
U_{c}^{v} \\
U_{o}^{\prime v}
\end{array}\right] .
$$

From the second row of $(24)$ we know that $U^{\prime v}{ }_{0}=$ $-\overline{\mathcal{A}}_{o o}^{v,-1} \overline{\mathcal{A}}_{o c}^{v} U_{c}^{v}$. Therefore, from the first row of (24) we get $\bar{I}_{c}^{v}=\left(\overline{\mathcal{A}}_{c c}^{v}-\overline{\mathcal{A}}_{c o}^{v} \overline{\mathcal{A}}_{o o}^{v,-1} \overline{\mathcal{A}}_{o c}^{v}\right) U_{c}^{v}$. Consequently, for a given $v$, the current/voltage model is

$$
\begin{aligned}
& \bar{I}=S^{v,-1} \mathcal{L}^{v} S^{v} U \\
& \mathcal{L}^{v}=\left[\begin{array}{cr}
\mathcal{A}_{c}^{v} & 0 \\
0 & 0
\end{array}\right] \\
& \mathcal{A}_{c}^{v} \triangleq \overline{\mathcal{A}}_{c c}^{v}-\overline{\mathcal{A}}_{c o}^{v} \overline{\mathcal{A}}_{o o}^{v,-1} \overline{\mathcal{A}}_{o c}^{v}
\end{aligned}
$$

where $\mathcal{A}_{c}^{v} \in \mathbb{R}^{m_{c, v} \times m_{c, v}}$ is the Schur complement matrix of the conductivity matrix $\overline{\mathcal{A}}$ and it represents the voltage/current relation of partially connected resistor network corresponding to the switch combination $v$.

We will denote by $\mathcal{R}^{v}$ the set of states $X$ for which a switch combination $v$ is feasible. The regions $\mathcal{R}^{v}$ are polyhedra defined by the diode properties

$$
\mathcal{R}^{v} \triangleq\left\{X: I_{o}^{v}=0, U_{o}^{v}-{U^{\prime}}_{o}^{v} \geq 0, I_{c}^{v} \geq 0\right\} .
$$

The conditions in (26) can be rewritten as a set of equalities and inequalities in the $X$ variable by recalling from (25) that $U=\left(\mathbb{I}^{m} \otimes\left[\begin{array}{ll}1 & 0\end{array}\right]\right) X$ and $\bar{I}=S^{v,-1} \mathcal{L}^{v} S^{v} U$

$$
\begin{aligned}
& \mathcal{R}^{v} \triangleq\left\{X:\left[0 \mathbb{I}^{m_{o}}\right] \mathcal{L}^{v} S^{v}\left(\mathbb{I}^{m} \otimes\left[\begin{array}{ll}
1 & 0
\end{array}\right]\right) X=0,\right. \\
&\left(\left[\begin{array}{lll}
0 & \left.\mathbb{I}^{m_{o}}\right]+\left(\overline{\mathcal{A}}_{o o}^{v,-1} \overline{\mathcal{A}}_{o c}^{v}\right)\left[\mathbb{I}^{m_{c}}\right. & 0
\end{array}\right]\right) S^{v}\left(\mathbb{I}^{m} \otimes\left[\begin{array}{ll}
1 & 0
\end{array}\right]\right) X \geq 0
\end{aligned}
$$$$
\left.\left[\begin{array}{ll}
\mathbb{I}^{m_{c}} & 0
\end{array}\right] \mathcal{L}^{v} S^{v}\left(\mathbb{I}^{m} \otimes\left[\begin{array}{ll}
1 & 0
\end{array}\right]\right) X \geq 0\right\}
$$

where $\mathbb{I}^{m}$ is the identity matrix of size $m$.

Model (18) is then rewritten as the interconnection between $m$ decoupled systems and a coupling feedback as shown in Fig. 5. Using (18) and (25) the closed loop system can be written as

$$
\begin{aligned}
\frac{d X}{d t^{\prime}}=f(X)= & \left(\mathbb{I}^{m} \otimes F\right) X+\left(S^{v,-1} \mathcal{L}^{v} S^{v} \otimes E\right) X \\
& +\left[b_{1} \ldots b_{m}\right]^{T} \otimes\left[\begin{array}{c}
0 \\
\frac{R \gamma}{2}
\end{array}\right], X \in \mathcal{R}^{v}
\end{aligned}
$$

where $\otimes$ is the Kronecker product, $F$ describes the dynamics of a single system and $E$ is a constant matrix

$$
\begin{aligned}
& F \triangleq\left[\begin{array}{cc}
-1 & 1 \\
-\gamma & \frac{\gamma-\gamma \varepsilon}{2}
\end{array}\right], \quad \varepsilon \triangleq \frac{2}{K} \\
& E \triangleq\left[\begin{array}{ll}
1 & 0 \\
0 & 0
\end{array}\right] .
\end{aligned}
$$

Rewrite (28) as a piecewise affine system

$$
\begin{aligned}
& \dot{X}=f(X)=A^{v} X+B, X \in \mathcal{R}^{v} \\
& A^{v} \triangleq \mathbb{I}^{m} \otimes F+S^{v,-1} \mathcal{L}^{v} S^{v} \otimes E \\
& B \triangleq\left[b_{1} \ldots b_{m}\right]^{T} \otimes\left[\begin{array}{c}
0 \\
\frac{R \gamma}{2}
\end{array}\right]
\end{aligned}
$$

where the polyhedra $\mathcal{R}^{v}$ are defined in (27).

Lemma 1 (Continuity of $f(\cdot)$ ). The function $f(\cdot)$ in (30) is continuous on its domain.

Proof. In the interior of each region $\mathcal{R}^{v}$, the $f(\cdot)$ is affine and thus continuous. We need to prove continuity of $f(\cdot)$ on the borders of region $\mathcal{R}^{v}$. Let $v_{1}$ and $v_{2}$ be two switch combinations and consider the shared border $\mathcal{B}$ of regions $\mathcal{R}^{v_{1}}$ and $\mathcal{R}^{v_{2}}, \mathcal{B}=\mathcal{R}^{v_{1}} \cap \mathcal{R}^{v_{2}}$.

Recall that the only term of the dynamic model (30) that depends on $v$ is the term $S^{v,-1} \mathcal{L}^{v} S^{v} U$ where $\bar{I}=$ $S^{v,-1} \mathcal{L}^{v} S^{v} U$. We will prove that the current $\bar{I}$ on the set $\mathcal{B}$ is equal for switch combinations $v_{1}$ and $v_{2}, \bar{I}^{v_{1}}=\bar{I}^{v_{2}}$. Let $\mathcal{C}$ be the set of switches which have the same status in $v_{1}$ and $v_{2}$ and its complement $\overline{\mathcal{C}}=[0,|\mathcal{I}|] \backslash \mathcal{C}$. The switches in $\overline{\mathcal{C}}$ change their state in $\mathcal{B}$ and, therefore, necessarily satisfy diode switch conditions: $I_{i}=0$ and $U=U^{\prime}$.

Consider the switch combinations $v_{1}$ and the set $\overline{\mathcal{C}}$. From the definition of $\mathcal{B}$, the currents across the switches $\overline{\mathcal{C}}$ must be equal to zero, either because the switch is open in $v_{1}$ or because it is open in $v_{2}$. In addition, for such switches $U=U^{\prime}$ either because the switch is closed in $v_{1}$ or because it is closed in $v_{2}$. Also currents across open switches in $\mathcal{C}$ are zero both in $v_{1}$ and $v_{2}$. Similarly $U=U^{\prime}$ for closed switches in $\mathcal{C}$. The same arguments can be repeated for the switch combination $v_{2}$. In conclusion, if we partition the currents $\bar{I}^{v_{1}}$ and $\bar{I}^{v_{2}}$ as

$$
\begin{gathered}
\bar{I}^{v_{1}}=\left[\bar{I}_{c, \mathcal{C}}^{v_{1}, T}, \bar{I}_{o, \mathcal{C}}^{v_{1}, T}, \bar{I}_{\overline{\mathcal{C}}}^{v_{1}, T}\right]^{T} \\
\bar{I}^{v_{2}, T}=\left[\bar{I}_{c, \mathcal{C}}^{v_{2}, T}, \bar{I}_{o, \mathcal{C}}^{v_{2}}, \bar{I}_{\overline{\mathcal{C}}}^{v_{2}, T}\right]^{T},
\end{gathered}
$$

on the border $\mathcal{B}$ we have:

$$
\begin{aligned}
& \bar{I}_{\overline{\mathcal{C}}_{1}}^{v_{1}}=\bar{I}_{\overline{\mathcal{C}}}^{v_{2}}=0 \\
& \bar{I}_{o, \mathcal{C}}^{v_{1}}=\bar{I}_{o, \mathcal{C}}^{v_{2}}=0 \\
& U^{\prime v_{1}, \mathcal{C}}=U^{\prime v_{2}}=X_{c, \mathcal{C}} \\
& U^{\prime v_{1}}=U^{\prime}{ }^{v_{2}}=X_{\overline{\mathcal{C}}}{ }^{v_{\mathcal{C}}}=X_{\overline{\mathcal{C}}}
\end{aligned}
$$

where $X_{\square}$ denotes the state variables $U$ in $X$ indexed by $\square$. Rewrite (20) for switch $v_{1}$ as

$$
\left[\begin{array}{c}
\bar{I}_{c, \mathcal{C}}^{v_{1}} \\
\bar{I}_{o, \mathcal{C}}^{v_{1}} \\
\bar{I}_{\overline{\mathcal{C}}}^{v_{1}}
\end{array}\right]=\overline{\mathcal{A}}\left[\begin{array}{c}
U^{\prime}{ }^{v_{1}}, \mathcal{C} \\
U^{\prime}{ }^{v_{1}} \\
U^{\prime}{ }^{\prime}, \mathcal{C} \\
\overline{\mathcal{C}},
\end{array}\right]
$$


and for $v_{2}$ as

$$
\left[\begin{array}{c}
\bar{I}_{c, \mathcal{C}}^{v_{2}} \\
\bar{I}_{o, \mathcal{C}}^{v_{2}} \\
\bar{I}_{\overline{\mathcal{C}}}^{v_{2}}
\end{array}\right]=\overline{\mathcal{A}}\left[\begin{array}{c}
U^{\prime} v_{2} \\
U^{\prime}{ }^{\prime}, \mathcal{C} \\
o, \mathcal{C} \\
U^{\prime}{ }_{\overline{\mathcal{C}}}^{v_{2}}
\end{array}\right] .
$$

The linear systems (34) and (35) have $m$ equations and $2 m$ variables $\left(\bar{I}\right.$ and $\left.U^{\prime}\right)$. On the border $\mathcal{B}$, from $(33) m$ of the variables are fixed and equal for both switch combinations from (33). The solution of $\bar{I}_{c, \mathcal{C}}^{v_{2}}$ and $\bar{I}_{c, \mathcal{C}}^{v_{1}}$ (and $U_{o, \mathcal{C}}^{\prime v_{1}}, U^{\prime v_{2}, \mathcal{C}}$ ) will yield the same value, thus proving the lemma.

\subsection{Global Exponential Stability}

Assumption 1. The circuit in Figure $4 b$ has a unique equilibrium point.

As shown in [17], the circuit has a unique equilibrium that corresponds to the solution of (1) for an ideal negative resistance $(\varepsilon \rightarrow 0)$. Therefore, in the limit $\varepsilon \rightarrow 0$ the assumption is equivalent to an assumption that (1) is feasible and admits a unique solution.

Theorem 1 (Global exponential stability). Let Assumption 1 hold. For any $\varepsilon>0$ there exists $\gamma_{\text {crit }}>0$ such that for any $\gamma, 0<\gamma<\gamma_{\text {crit }}$ the equilibrium of the optimization circuit in Figure $4 b$ is globally exponentially stable.

We prove the theorem using a piecewise quadratic Lyapunov function. The derivative of the Lyapunov function is proved to be negative definite using an eigenvalue decomposition of the resistance matrix. This Lyapunov function has the form (37) below due to Krasovskiu [12] (see also [11] and $[3])$.

Lemma 2 (Locally Lipschitz Lyapunov function). For the system defined in (30), if $A^{v}$ is not singular and there exists $\mathcal{P}=\mathcal{P}^{T}>0$ such that

$$
\mathcal{P} A^{v}+A^{v T} \mathcal{P} \prec 0, \forall v \in\left\{0, \ldots, 2^{|\mathcal{I}|}-1\right\},
$$

then

$$
L(X)=f(X)^{T} \mathcal{P} f(X)
$$

is a Lyapunov function and the equilibrium point is globally exponentially stable.

Proof. The function $L(X)$ equals zero only at equilibrium and positive elsewhere. The function $L(\cdot)$ is differentiable almost everywhere, except in a set of measure zero (region borders). From (36) we know that, for almost all $t$,

$$
\dot{L}(X(t))=f(X(t))^{T}\left(\mathcal{P} A^{v}+A^{v T} \mathcal{P}\right) f(X(t)) \leq-\alpha L(X(t)),
$$

where $\alpha>0$ is such that

$$
\mathcal{P} A^{v}+A^{v T} \mathcal{P} \leq-\alpha \mathcal{P}
$$

The function $L(X(t))$ is a locally Lipschitz continuous function of $X(t)$. $X(t)$ is absolutely continuous since it is the solution of the ordinary differential equation (28) [2]. Therefore, $L(X(t))$ is an absolutely continuous function of $t$, and since (38) holds for almost all $t$,

$$
L(X(t)) \leq L\left(X\left(t_{0}\right)\right) e^{-\alpha\left(t-t_{0}\right)}, \quad \forall t>t_{0}
$$

and the exponential stability trivially follows from (40). Global stability is achieved since the Lyapunov function (37) is radially unbounded because both $\mathcal{P}$ and $A^{v}$ are non singular from the lemma assumptions.

Lemma 3 (Existence of Lyapunov matrix). Let $A^{v}$ be as defined in (30b), where $\mathcal{L}^{v}=\mathcal{L}^{v T}$ as defined in (25b), and $\lambda_{v j}\left(\mathcal{L}^{v}\right) \in\left[\lambda_{\min }, 0\right]$ is the $j$-th eigenvalue of $\mathcal{L}^{v}$, and $F, E$ as defined in (29). If there exists $P$ such that

$$
P(F+\lambda E)+(F+\lambda E)^{T} P \prec 0
$$

for all $\lambda \in\left[\lambda_{\min }, 0\right]$, then $\mathcal{P}=\mathbb{I}^{m} \otimes P$ satisfies $(36)$.

Proof. Define $T \in \mathbb{R}^{m_{c} \times m_{c}}, T^{T} T=\mathbb{I}^{m}$ to be a matrix whose $j$-th column is an eigenvector corresponding to $\lambda_{v j}\left(S^{v,-1} \mathcal{L}^{v} S^{v}\right)$. Then $T^{v,-1} S^{v,-1} \mathcal{L}^{v} S^{v} T^{v}=\Lambda^{v}$ diagonalizes $S^{v,-1} \mathcal{L}^{v} S^{v}$. Motivated by an observation that the variable transformation $Z=\left(T^{v,-1} \otimes \mathbb{I}^{2}\right) X$ diagonalizes the system, we multiply the matrix $\left(\mathcal{P} A^{k}+A^{v^{T}} \mathcal{P}\right)$ from (38) by this transformation from left and right and use the definition of $A^{v}$ and $\mathcal{P}$ to get

$$
\begin{aligned}
&\left(T^{v,-1} \otimes \mathbb{I}^{2}\right)\left(\mathcal{P} A^{v}+A^{v T} \mathcal{P}\right)\left(T^{v} \otimes \mathbb{I}^{2}\right) \\
&=\left(T^{v,-1} \otimes \mathbb{I}^{2}\right)\left[\left(\mathbb{I}^{m} \otimes P\right)\left(\mathbb{I}^{m} \otimes F+S^{v,-1} \mathcal{L}^{v} S^{v} \otimes E\right)\right. \\
&\left.+\left(\mathbb{I}^{m} \otimes F+S^{v,-1} \mathcal{L}^{v} S^{v} \otimes E\right)^{T}\left(\mathbb{I}^{m} \otimes P\right)\right]\left(T^{v} \otimes \mathbb{I}^{2}\right) \\
&=\left(T^{v,-1} \otimes \mathbb{I}^{2}\right)\left[\mathbb{I}^{m} \otimes\left(P F+F^{T} P\right)+\right. \\
&\left.+S^{v,-1} \mathcal{L}^{v} S^{v} \otimes\left(P E+E^{T} P\right)\right]\left(T^{v} \otimes \mathbb{I}^{2}\right) \\
&= \mathbb{I}^{m} \otimes\left(P F+F^{T} P\right)+\Lambda^{v} \otimes\left(P E+E^{T} P\right) .
\end{aligned}
$$

This is a block diagonal matrix where each block is given by $P\left(F+\lambda_{v j} E\right)+\left(F+\lambda_{v j} E\right)^{T} P$. Since this block is a negative definite matrix according to (41), the matrix $\mathcal{P}$ ensures $\mathcal{P} A^{v}+A^{v T} \mathcal{P} \prec 0$ for all $v \in\left[0,2^{|\mathcal{I}|}-1\right]$.

Lemma 4 (Eigenvalues of $\overline{\mathcal{A}}$ ). The eigenvalues of

$$
\overline{\mathcal{A}}=\bar{A} \operatorname{diag}\left(\mathbf{1}^{T} \bar{A}\right)^{-1} \bar{A}^{T}-\mathbb{I}^{m}
$$

are in $[-1,0]$.

Proof. The matrix $\overline{\mathcal{A}}$ is negative semi-definite (see $[17$, Lemma 3]). The matrix $\bar{A} \operatorname{diag}\left(\mathbf{1}^{T} \bar{A}\right)^{-1} \bar{A}^{T}=C C^{T}$ is positive semi-definite, where $C=\bar{A} \operatorname{diag}\left(\mathbf{1}^{T} \bar{A}\right)^{-1 / 2}$. Therefore, eigenvalues of $\bar{A} \operatorname{diag}\left(\mathbf{1}^{T} \bar{A}\right)^{-1} \bar{A}^{T}$ are non-negative. Consequently, eigenvalues of $\bar{A} \operatorname{diag}\left(\mathbf{1}^{T} \bar{A}\right)^{-1} \bar{A}^{T}-\mathbb{I}^{m}=\overline{\mathcal{A}}$ are greater or equal then -1 .

Lemma 5 (Eigenvalues of $\mathcal{A}_{c}$ ). The eigenvalues of

$$
\mathcal{A}_{c}=\overline{\mathcal{A}}_{c c}-\overline{\mathcal{A}}_{c o} \overline{\mathcal{A}}_{o o}^{-1} \overline{\mathcal{A}}_{o c}
$$

are in $[-1,0]$ for any partitioning of $\overline{\mathcal{A}}$. 
Proof. The matrix $\mathcal{A}_{c}$ is a Schur complement of the block $\overline{\mathcal{A}}_{o o}$ in the symmetric matrix $\overline{\mathcal{A}}$. Therefore, the eigenvalues of $\mathcal{A}_{c}$ are bounded between -1 and 0 , since eigenvalues of a Schur complement matrix [14] are bounded by the eigenvalues of the original matrix $\overrightarrow{\mathcal{A}}$.

For the next lemma we decompose the matrix $E$ as

$$
E=\left[\begin{array}{ll}
1 & 0 \\
0 & 0
\end{array}\right]=\left[\begin{array}{l}
1 \\
0
\end{array}\right]\left[\begin{array}{ll}
1 & 0
\end{array}\right] \triangleq B C .
$$

Lemma 6 (Existence of $\mathrm{P})$. There exists a matrix $P=$ $P^{T}>0$ satisfying

$$
P(F+\lambda B C)+(F+\lambda B C)^{T} P<0 \quad \forall \lambda \in[-1,0]
$$

if and only if there exists $\tilde{P}=\tilde{P}^{T}>0$ such that

$$
\left[\begin{array}{cc}
\tilde{P} F+F^{T} \tilde{P} & \tilde{P} B-C^{T} \\
B^{T} \tilde{P}-C & -2
\end{array}\right]<0 .
$$

Proof. We first rewrite (44) as:

$$
x^{T}\left[P(F+\lambda B C)+(F+\lambda B C)^{T} P\right] x<0
$$

$\forall x \neq 0, \forall \lambda \in[-1,0]$. Next, define $y \triangleq \lambda C x$ and write (46) as:

$$
\left[\begin{array}{l}
x \\
y
\end{array}\right]^{T}\left[\begin{array}{cc}
P F+F^{T} P & P B \\
B^{T} P & 0
\end{array}\right]\left[\begin{array}{l}
x \\
y
\end{array}\right]<0 .
$$

Since $\lambda \in[-1,0]$ and $y=\lambda C x$, we get

$$
y C x \leq-y^{2}
$$

that is

$$
\left[\begin{array}{l}
x \\
y
\end{array}\right]^{T}\left[\begin{array}{cc}
0 & -C^{T} \\
-C & -2
\end{array}\right]\left[\begin{array}{l}
x \\
y
\end{array}\right] \geq 0 .
$$

Thus, (44) means that (47) holds for all $x \neq 0, y \neq 0$ restricted by (49).

We now invoke the $S$-procedure [1] which states that, for symmetric matrices $T_{0}, T_{1}$,

$$
\zeta^{T} T_{0} \zeta<0 \quad \text { for all } \zeta \neq 0 \text { satisfying } \quad \zeta^{T} T_{1} \zeta \geq 0
$$

if there exists $q_{1}>0$ such that

$$
T_{0}+q_{1} T_{1}<0
$$

Since the matrices in (47) and (49) play the role of $T_{0}$ and $T_{1}$ respectively, we conclude from the S-procedure that (44) holds if:

$$
\left[\begin{array}{cc}
P F+F^{T} P & P B-q_{1} C^{T} \\
B^{T} P-q_{1} C & -2 q_{1}
\end{array}\right]<0
$$

for some $q_{1}>0$. To prove the "if" part of the lemma, we take the $\tilde{P}$ satisfying (45), substitute $P=\tilde{P}$ and $q_{1}=1$ in (52), and conclude that (44) holds with $P=\tilde{P}$.
To prove the "only if" part, we recall that the S-procedure states that (50) also implies (51) for some $q_{1} \geq 0$, provided there exists $\zeta_{0}$ such that $\zeta_{0}^{T} T_{1} \zeta_{0}>0$ [1]. Selecting an $x_{0}$ such that $C x_{0} \neq 0$, we confirm that $\zeta_{0} \triangleq\left[x_{0}^{T}-\frac{1}{2} C x_{0}\right]^{T}$ satisfies $\zeta_{0}^{T} T_{1} \zeta_{0}>0$ for the matrix $T_{1}$ in (49). Thus, (44) implies (52) for some $q_{1} \geq 0$; in fact, $q_{1}>0$ since the matrix in (52) cannot be negative definite with $q_{1}=0$. It then follows that (45) holds with the choice $\tilde{P}=\frac{1}{q_{1}} P$.

Lemma 7 (KYP). For any value of $\varepsilon>0$ there exists $\gamma_{\text {crit }}>0$ such that for any $0<\gamma<\gamma_{\text {crit }}$ there exists $\tilde{P}$ satisfying (45).

Proof. It follows from the Kalman-Yakubovich-Popov (KYP) Lemma [15] that there exists $\tilde{P}>0$ satisfying (45) if

$$
\begin{aligned}
& {\left[\begin{array}{c}
\left(j \omega I_{2}-F\right)^{-1} B \\
1
\end{array}\right]^{*}\left[\begin{array}{cc}
0 & -C^{T} \\
-C & -2
\end{array}\right]\left[\begin{array}{c}
\left(j \omega I_{2}-F\right)^{-1} B \\
1
\end{array}\right]=} \\
& \frac{2\left(1-\varepsilon^{2}\right) \gamma^{2}-8 \omega^{2}}{4 \omega^{4}+\omega^{2}\left((\varepsilon-1)^{2} \gamma^{2}-8 \gamma+4\right)+(\varepsilon+1)^{2} \gamma^{2}}-2<0, \forall \omega \in \mathbb{R} .
\end{aligned}
$$

When $\omega^{2} \rightarrow \infty$, (53) converges to -2 . The expression (53) can yield positive values either by continuously crossing zero or when the fraction denominator vanishes while the expression is negative and causes a leap via $-\infty$ to a positive value.

The zero crossing can be checked from

$$
8 \omega^{4}+\left(2(\varepsilon-1)^{2} \gamma^{2}-16 \gamma+16\right) \omega^{2}+4 \varepsilon^{2} \gamma^{2}+4 \varepsilon \gamma^{2}=0 .
$$

This is a polynomial in $\omega^{2}$ and has no roots with positive real part $\left(\omega^{2}<0\right)$ if all the coefficients have the same sign, according to Routh-Hurwitz criterion for second order polynomials. The last term $4 \varepsilon^{2} \gamma^{2}+4 \varepsilon \gamma^{2}$ is positive for $\varepsilon>0$ and $\gamma>0$. It is immediate to see that the second term is positive for a small $\gamma$

$$
\gamma<\frac{4-2 \sqrt{4-2(\varepsilon-1)^{2}}}{(\varepsilon-1)^{2}}=\gamma_{\text {crit },(55)} .
$$

The denominator in (53) is a second order polynomial in $\omega^{2}$ as well and we can bound $\gamma$ in a similar manner using Routh-Hurwitz criterion which yields

$$
\gamma<\frac{4-2 \sqrt{4-(\varepsilon-1)^{2}}}{(\varepsilon-1)^{2}}=\gamma_{\text {crit },(56)} .
$$

Thus, there is no $\omega$ that cause the denominator to vanish if $\gamma$ satisfies (56). Therefore, for a $\gamma$ satisfying (55) and (56) there is no real $\omega$ that can make (53) positive, consequently, there exists $\gamma_{\text {crit }}$ such that $0<\gamma<\gamma_{\text {crit }}$ satisfies (45).

Note that the criteria (55) and (56) are conservative, since Routh-Hurwitz criterion excludes not only real positive roots but complex positive roots as well. A tight bound for $\gamma_{\text {crit }}$ may be obtained when we exclude imaginary roots by examining the discriminant of (54) and the discriminant of the denominator in (53). As can be seen 


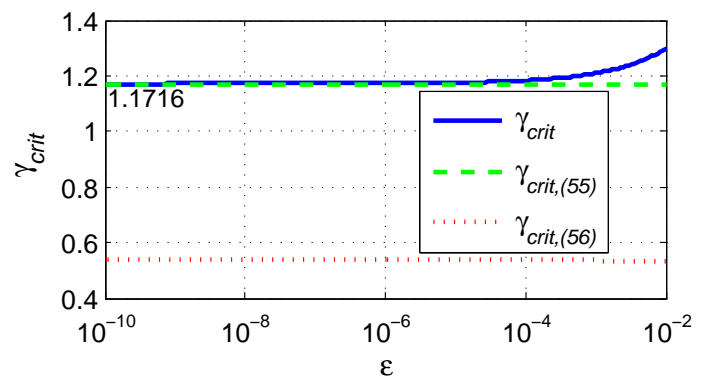

Figure 6: The tight bound $\gamma_{\text {crit }}$, and the conservative bounds $\gamma_{\text {crit },(55)}$ and $\gamma_{\text {crit,(56) }}$ vs $\varepsilon$ as in Lemma 7.

in Fig. 6, $\gamma_{\text {crit }}$ approaches 1.1716 as $\varepsilon \rightarrow 0$ and admits larger values for a larger $\varepsilon$. Note that high values of $\varepsilon$ are not of interest, since high gain opamps are required for reasonable accuracy. In fact, a typical opamp gain is of the order of $10^{3}$ to $10^{6}$.

Remark 1. The existence of the matrix $P$ as required in Lemma 3 can be alternatively shown by numerically checking feasibility as function of $\gamma$ and $\varepsilon$ of the LMI

$$
\begin{aligned}
& P \succ 0 \\
& F^{T} P+P F+\lambda_{i}(E P+P E) \prec 0, \quad \lambda_{i}=-1,0
\end{aligned}
$$

where $F$ is a function of $\gamma$ and $\varepsilon$ as in (29).

The following lemma is required to prove that the stability is global.

Lemma 8 (Nonsingularity of $A^{v}$ ). If $\gamma<2$, the matrix $A^{v}$ in $(30 \mathrm{~b})$ is non-singular for all $v \in\left[0,2^{|\mathcal{I}|}-1\right]$.

Proof. From (30b) the eigenvalues of $A^{v}$ are bounded by the sum of eigenvalues of $I_{m} \otimes F$ and $S^{v,-1} \mathcal{L}^{v} S^{v} \otimes E$. The later eigenvalues belong to $[-1,0]$ as from Lemma 5 . The eigenvalues of $F$ are strictly negative for $\gamma<(2+\varepsilon)<$ 2 , as can be easily shown using (29) and Routh-Hurwitz stability criterion. Therefore, the eigenvalues of $A^{v}$ are strictly negative for $\gamma<2$.

Proof of Theorem 1: We complete the proof of Theorem 1 by assembling the intermediate results. Lemma 7 states that there exists $\tilde{P}$ for a correct range of $\gamma$ such that (45) holds. Then from Lemma 6 we conclude that there exists $P$ such that (44) holds, and, thus, Lemma 3 holds with $\lambda_{\text {min }}=-1$ as from Lemmas 4 and 5 . Lemma 8 holds for any $\gamma<2\left(\gamma_{\text {crit }}\right.$ is actually bounded to a lower values as in Fig. 6). Lemmas 3 and 8 yield the conditions for Lemma 2 to hold and this completes the proof.

\section{Conclusion}

We have proven that the $\mathrm{QP}$ optimization circuit is exponentially stable if $\gamma=\frac{R C K}{\tau}<\min \left(2, \gamma_{\text {crit }}\right)$. The gain-bandwidth-product (GBWP) of an opamp is given by $\frac{2 \pi K}{\tau}$ and the stability condition may be interpreted as
GBWP $<\frac{2 \pi \gamma_{\text {crit }}}{R C}$, meaning that the closed loop time constant of opamp is limited by the $R C$ time constant.

When the circuit is implemented in a nanoscale using analog VLSI technology with $R \simeq 1 \mathrm{~K} \Omega$ and $C \simeq 10 \mathrm{fF}$ $\left(R C \simeq 10^{-12} \mathrm{sec}\right)$, the gain-bandwidth-product is limited by roughly $120 \mathrm{GHz}$. For a larger implementation on a printed circuit board (PCB) with $R \simeq 10 \mathrm{~K} \Omega$ and $C \simeq 100 \mathrm{pF}$, the gain-bandwidth-product is limited by 1.2 MHz. Therefore, the circuit should achieve nanosecond range convergence time if implemented using high speed technology, such as analog VLSI.

\section{References}

[1] Boyd, S., El Ghaoui, L., Feron, E., Balakrishnan, V., 1994. Linear Matrix Inequalities in System and Control Theory. Studies in Applied Mathematics. Society for Industrial and Applied Mathematics.

[2] Coddington, E. A., Levinson, N., 1955. Theory of ordinary differential equations. Tata McGraw-Hill Education.

[3] Coogan, S., Arcak, M., Jul. 2013. A note on norm-based Lyapunov functions via contraction analysis. ArXiv e-prints.

[4] Dennis, J. B., 1959. Mathematical programming and electrical networks. Technology Press of the Massachusetts Institute of Technology [Cambridge].

[5] Ferrari-Trecate, G., Cuzzola, F. A., Mignone, D., Morari, M., 2002. Analysis of discrete-time piecewise affine and hybrid systems. Automatica 38 (12), 2139 - 2146.

[6] Garcia, C. E., Prett, D. M., Morari, M., 1989. Model predictive control: theory and practice - a survey. Automatica 25 (3), 335348.

[7] Hassibi, A., Boyd, S., Jun 1998. Quadratic stabilization and control of piecewise-linear systems. In: American Control Conference, 1998. Proceedings of the 1998. Vol. 6. pp. 3659-3664 vol.6.

[8] Heemels, W. P. M. H., Camlibel, M., Schumacher, J., 2002. On the dynamic analysis of piecewise-linear networks. Circuits and Systems I: Fundamental Theory and Applications, IEEE Transactions on 49 (3), 315-327.

[9] Johansson, M., Rantzer, A., 1998. Computation of piecewise quadratic lyapunov functions for hybrid systems. IEEE transactions on automatic control 43 (4), 555-559.

[10] Kennedy, M., Chua, L., may 1988. Neural networks for nonlinear programming. Circuits and Systems, IEEE Transactions on $35(5), 554-562$.

[11] Khalil, H., 2002. Nonlinear Systems. Prentice Hall PTR.

[12] Krasovskiu, N. N., 1963. Stability of motion: applications of Lyapunov's second method to differential systems and equations with delay. Stanford University Press.

[13] Lin, H., Antsaklis, P. J., 2009. Stability and stabilizability of switched linear systems : A survey of recent results. IEEE Transactions on Automatic control 54 (2), 308-322.

[14] Liu, J., Huang, Y., Liao, A., 2006. Some inequalities for eigenvalues of schur complements of hermitian matrices. Journal of Computational and Applied Mathematics 196 (2), 439 - 451.

[15] Rantzer, A., 1996. On the kalman-yakubovich-popov lemma. Systems \& Control Letters 28 (1), $7-10$.

[16] Tank, D., Hopfield, J., may 1986. Simple 'neural' optimization networks: An a/d converter, signal decision circuit, and a linear programming circuit. Circuits and Systems, IEEE Transactions on $33(5), 533-541$.

[17] Vichik, S., Borrelli, F., 2014. Solving linear and quadratic programs with an analog circuit. Computers \& Chemical Engineering. 